\title{
Pancreatic tuberculosis in immunocompetent patients
}

\author{
At the University Hospital in Kingdom of Saudi Arabia
}

Faisal A. Alsaif, MD, FRCSC, Mazen M. Hassanain, MD, PhD, Abdulrahman M. Aljebreen, MD, Abdulsalam B. Alsharaabi, MD, Faisal A. Alalem, MD, Reem A. Alharbi, MD, Fatima S. Ali, MBBS, Hend K. Idriss, MD, Ola A. Alkhalaf, MBBS.

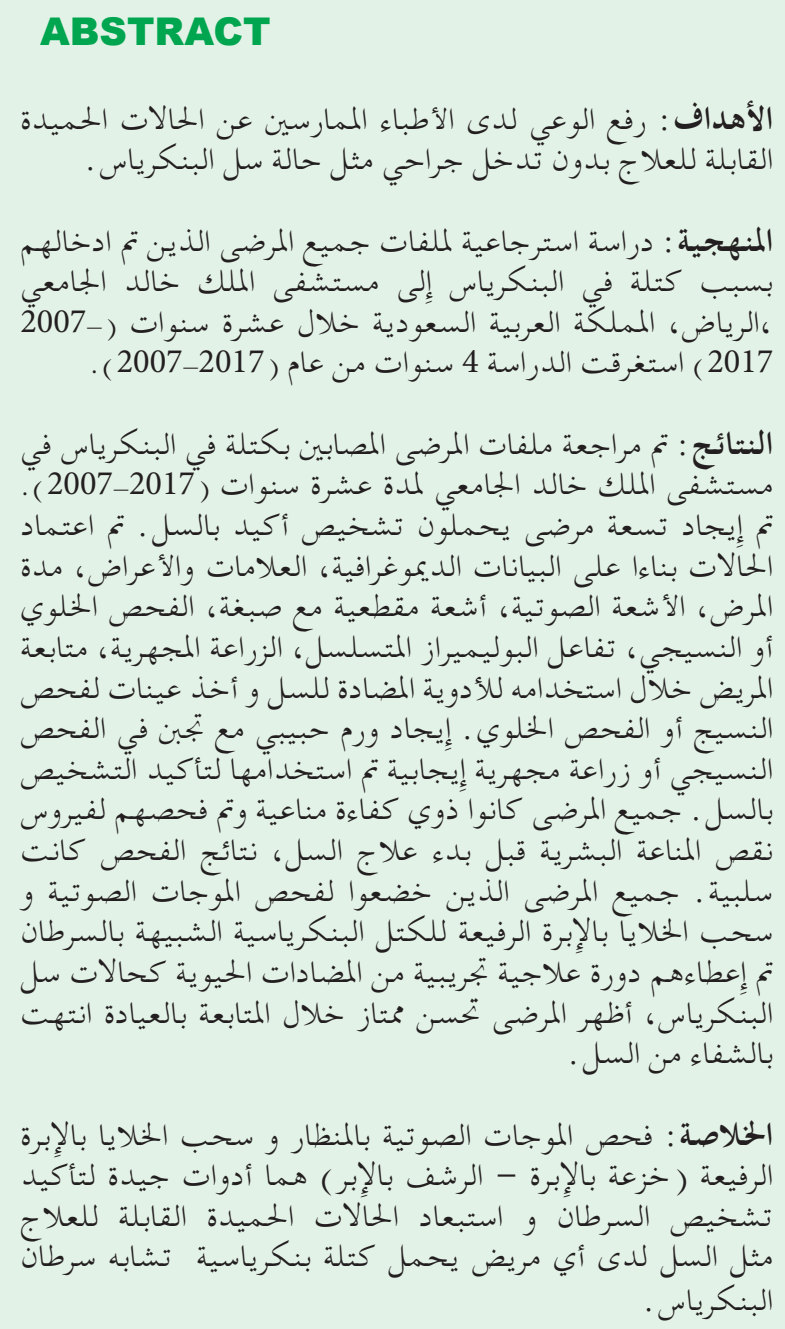

Objectives: To raise awareness of practitioners on benign treatable conditions such as pancreatic tuberculosis (TB).

Methods: A retrospective study at King Khaled University Hospital, Riyadh, Saudi Arabia of all patient charts presented with pancreatic mass for a period of 10 years (2007-2017) with a study duration of 4 years between 2013 and 2017. Patients with confirmed diagnosis of pancreatic cancer were excluded. A written ethical approval was obtained accordingly.

Results: All adult patient charts were retrospectively reviewed with a pancreatic mass for a period of 10 years (2007-2017). Nine patients were identified with proven diagnosis of TB. The data were obtained based on demographic features, sign and symptoms, duration of illness, imaging, ultrasound, contrast enhanced computed tomography scan, cytology or histopathology, polymerase chain reaction, culture and follow up with anti-tuberculous therapy and samples for cytology or histology. The histological findings of granuloma with caseation or positive culture were used confirming the diagnosis of TB. All patients were immunocompetent and screened for human immunodeficiency viruses before starting anti-TB treatment. Results were negative. All patients who underwent fine needle aspiration (FNA) and endoscopic ultrasound (EUS) for suspicious pancreatic mass were provided trial of antibiotics as cases of pancreatic tuberculosis showed dramatic improvement during follow up and cured from the disease.

Conclusion: The EUS and FNA are good tools to confirm malignancy and rule out benign treatable conditions like TB for any patient with a pancreatic mass suspicious for carcinoma.

$$
\text { Saudi Med J 2019; Vol. } 40 \text { (10): 1052-1057 }
$$$$
\text { doi: 10.15537/smj.2019.10.24245 }
$$

From the Department of Surgery (Alsaif, Hassanain, Alsharaabi, Alalem, Idriss), from Department of Medicine (Aljebreen), College of Medicine, King Saud University; from the Clinical Science Department (Alharbi), Princess Nourah Bint Abdul Rahman University; and from the Department of Medicine, (Ali, Alkhalaf), Almaarefa University, Riyadh, Kingdom of Saudi Arabia.

Received 19th May 2019. Accepted 8th August 2019.

Address correspondence and reprint request to: Dr. Faisal A. Alsaif, Associate Professor of Surgery and Consultant, HPB and Transplant Surgery, Department of Surgery, College of Medicine, King Saud University, Riyadh, Kingdom of Saudi Arabia. E-mail: falsaif1972@gmail.com

ORCID ID: orcid.org/0000-0002-2522-1681 
$\mathrm{T}$ uberculosis (TB) is a health problem arising mostly in immunocompromised individuals. There has been an elevated progression of cases reported in immunocompetent patients, more typical in developing countries. ${ }^{1}$

Pancreatic TB is extremely a rare infection and usually present clinically and radiologically similar to pancreatic adenocarcinoma. Furthermore, the actual transmission of the virus to the pancreas is yet unknown, but there are several approaches that have been proposed. It can be via blood-borne or lymphatic spreading from an occult lesion or invasion from contiguous lymph nodes or from an occult focus in the lungs. ${ }^{1}$ The practice of using endoscopic ultrasound (EUS), histology and cytology evaluation of pancreatic lesions are an advantage to classify pancreatic $\mathrm{TB}$, preventing repeated surgical interventions with major morbidity and mortality.

The aim of this study is to Raise awareness of practitioners about benign treatable conditions such as Pancreatic Tuberculosis.

Methods. A retrospective review on charts of all pancreatic mass patients presented at King Khaled University Hospital, Riyadh, Saudi Arabia for over 10 years (2007-2017) with a study duration of 4 years from 2013 to 2017. Nine patients were found positive of TB. Demographic information, abnormalities, illness duration, diagnostic tests (chest x-ray, ultrasound [US], contrast enhanced computed tomography [CT] scan), cytology or histopathology, PCR (polymerase chain reaction), culture and intervention data on anti-TB therapy were gathered. Cytological or histological specimen results had been collected through US guided fine needle aspiration (FNA). Diagnosis of TB had been confirmed based on the histopathological results of granuloma with caseation or positive culture. All patients are immunocompetent (human immunodeficiency viruses [HIV] screened, before starting anti-TB treatment, and results were negative). The references search method was conducted through online browsing. The study is in accordance with Helsinki Declaration. Ethical approval was obtained

Case Report. Case 1. A 44-year-old Saudi male, known case of hyperlipidemia, presented with a 2-year

Disclosure. Authors have no conflict of interests, and the work was not supported or funded by any drug company. history of fever, night sweats, abdominal pain and lethargy. Physical examination was unremarkable. Chest x-ray was normal. Computed tomography scan of the abdomen showed multiloculated cystic lesion at the tail of pancreas with multiple mediastinal lymph nodes. Endoscopic ultrasound and FNA were performed and showed necrotizing granulomatous inflammation.

Case 2. A 53-year-old Saudi male came with a one year history of upper abdominal pain, anorexia and weight loss. He had a history of hemoptysis. Physical examination was unremarkable. Chest x-ray showed multiple nodules. Computed tomography abdomen revealed a hypodense mass in the body of the pancreas with multiple enlarged lymph nodes, with 2 pulmonary nodules. Endoscopic ultrasound with FNA showed necrotizing granulomatous inflammation. Additional surgical biopsy was taken from a peritoneal nodule that showed granulomatous inflammation (disseminated TB).

Case 3. A 43-year-old Filipino male presented with nausea, vomiting and jaundice. Physical examination was unremarkable. Chest x-ray was normal. Ultrasound showed a dilated common bile duct. Endoscopic retrograde cholangiopancreatography (ERCP) was carired out and showed a bile duct with a stricture, a stent was inserted. Computed tomography abdomen showed multiple lymph nodes in porta hepatitis and peripancreatic area. Endoscopic ultrasound was performed and FNA from one of the peripancreatic lymph nodes revealed necrotizing granulomatous inflammation. Positive culture for TB (disseminated TB).

Case 4. A 21-year-old Saudi female admitted for abdominal pain, weight loss, vomiting, night sweats and fever for 2 months. She reported exposure to a TB patient 8 months before admission. On examination, there was tenderness in the epigastric area, with a small mass measuring $2 \times 3 \mathrm{~cm}$ in the upper abdomen. The mass was tender, well defined, hard, and deep. Patient had no peripheral lymphadenopathy and no organomegaly. Chest $\mathrm{x}$-ray was normal. Computed tomography scan of the abdomen showed peripancreatic hypodense cystic lesions. Endoscopic ultrasound and FNA was carried out and the biopsy showed necrotizing granulomatous inflammation and the PCR test came positive for TB.

Case 5. A 52-year-old Saudi female came with a 6 month history of upper abdominal pain associated with vomiting and weight loss. Physical examination was unremarkablea. Chest x-ray was normal. Computed tomography scan of the abdomen showed an ill-defined heterogeneous mass in the head and neck of pancreas 
invading the major vessels with multiple enlarged lymph nodes. The lesions were cystic in nature. Endoscopic ultrasound was carried out with FNA and did not show granulomas but mucin was positive. She was diagnosed as inoperable cystadenocarcinoma of pancreas. Tuberculosis culture came back later as positive.

Case 6. A 65-year-old Saudi female presented with abdominal pain for 3 years. On examination, a left cervical lymph nodes was palpable, tenderness in the epigastric area were noted. Chest $\mathrm{x}$-ray was normal. Computed tomography scan showed a pancreatic head mass with multiple enlarged lymph nodes, and multiple mediastinal lymph nodes. Endoscopic ultrasound was carried out with FNA and showed necrotizing granulomatous inflammation (disseminated TB).

Case 7. A 15-year-old Saudi boy presented with fever, shortness of breath, abdominal pain and loss of weight. On examination, he had peripheral cyanosis and nail clubbing. Chest $\mathrm{x}$-ray showed increased broncho-vesicular marking with prominent hilar shadows. Computed tomography chest revealed 2 small end vascular pulmonary nodules in the right upper and lower lobe, multiple mediastinal and hilar lymph nodes of variable sizes. Computed tomography abdomen revealed multiple abdominal lymph nodes in the gastro-splenic, porta hepatis, and near the pancreatic head with larger lymph node at the gastro-splenic area measuring approximately $4.7 \times 4 \mathrm{~cm}$. Endoscopic ultrasound with FNA showed multiple matted, large paraortic and celiac lymphadenopathy, suggestive of disseminated TB.

Case 8. A 59-year-old male, known case of chronic pancreatitis presented with recurrent abdominal pain, vomiting for one month and weight loss. On examination, abdomen was mildly tender in epigastric area, liver was palpable (liver span $14 \mathrm{~cm}$ ), no splenomegaly or ascites. Chest $\mathrm{x}$-ray showed fibroatelectatic changes in both lower zones. Computed tomography chest revealed bilateral lobe consolidation and left pleural effusion. Computed tomography abdomen scan showed a heterogeneous mass at the head of the pancreas with classification; and lymph nodes were noted at porta hepatis. Endoscopic ultrasound showed a solid mass with cystic component and FNA confirmed granulomatous inflammation.

Case 9. A 28-year-old Saudi male presented with a mass in peripancreatic area, discovered incidentally during pre-operative investigation of laparoscopic sleeve gastrectomy operation. He had abdominal pain only. Computed tomography chest, abdomen, pelvis showed upper abdominal and mediastinal lymphadenopathy along with peripancreatic homogeneously hypovascular soft tissue lesion residing in the gastrohepatic area, encasing the celiac trunk branches as well as main portal vein which was however patent. Endoscopic US was carried out with FNA from celiac lymph nodes showed necrotizing granulomatous lymphadenitis (disseminated TB).

The patients' demographics, symptoms, radiological findings, culture and presence of extra pancreatic TB are summarized in Table 1.

Management and results. All patients underwent anti-TB treatment after discussion with infection diseases team and the aim was to treat according to the National Tuberculosis Control and Prevention Program (NTCPP) guidelines.

Case 1. Patient started for 6 months on isonicotinylhydrazide (INH), ethambutol, pyridoxine, pyrazinamide, vitamin (vit) B6. Had hyperlipidemia, on atorvastatin which has interaction with rifampicin. Other alternatives were used. Followed up in the clinic, showed improvement.

Case 2. Patient started on isoniazid, rifampicin, ethambutol, pyrazinamide, vit B6. Followed up in the clinic for 9 months with improvement, then follow-ups were missed.

Case 3. Patient started on rifampicin, INH, pyrazinamide, ethambutol, pyridoxine, followed up in the clinic, showed improvement.

Case 4. Patient started on rifampicin, INH, pyrazinamide, ethambutol, pyridoxine. Followed up in the clinic, and showed improvement.

Case 5. Developed anti-TB medication toxicity, after starting the drugs (isoniazid, ethambutol, pyrazinamid). She developed drug-induced hepatitis and treatment was suspended until liver function test had improved. The drugs were started gradually again, and aspartate aminotransferase (AST) levels were measured as follow-up. After 2 months, she complained of fatigue, postural dizziness, heat intolerance and significant weight loss. As a result, the aim was to decrease the dose of Isoniazid. After 4 months, she reported pain and burning sensation in her left side of the body from head to toe, headache and dizziness. A neurological examination showed hyper-reflexia bilaterally in her lower limbs and power of $4 / 5$ bilaterally in all her body muscles, but no signs of meningeal irritation. Thus, rifampicin was added and isoniazid was stopped. Subsequently, she finished her treatment course with no major issues.

Case 6. Patient started on rifampicin, INH, pyrazinamide, ethambutol, pyridoxine, vit B6. Followed up in the clinic, showed improvement. 
Table 1 - Patient's demographics, symptoms, radiological findings, culture, and presence of extra pancreatic tuberculosis.

\begin{tabular}{|c|c|c|c|c|c|c|c|c|}
\hline No & Age & Gender & Clinical presentation & CT Findings & $\begin{array}{c}\text { Granulomatous } \\
\text { Inflammation }\end{array}$ & $\begin{array}{c}\mathrm{ZN} \\
\text { stain }\end{array}$ & $\begin{array}{l}\text { Culture of } \\
\text { M. TB }\end{array}$ & $\begin{array}{c}\text { Presence of extra } \\
\text { pancreatic TB }\end{array}$ \\
\hline 1 & 44 & $\mathrm{M}$ & $\begin{array}{l}\text { Abdominal pain, lethargy, } \\
\text { fever and night sweat }\end{array}$ & $\begin{array}{l}\text { Multiloculated cystic lesion with } \\
\text { thin enhancing in the tail of the } \\
\text { pancreases, multiple LN. }\end{array}$ & present & ve+ & ve- & 1 \\
\hline 2 & 53 & M & $\begin{array}{c}\text { Abdominal pain, weight loss, } \\
\text { anorexia }\end{array}$ & $\begin{array}{l}\text { Well-defined heterogeneously } \\
\text { hypodense lesion in the body of } \\
\text { pancreas, multiple LN. }\end{array}$ & present & ND & ND & $\begin{array}{l}\text { Chest, pancreas, LN, } \\
\text { peritoneal }\end{array}$ \\
\hline 3 & 43 & M & Jaundice & $\begin{array}{l}\text { Multiple necrotic LN in } \\
\text { peripancreatic and porta hepatitis }\end{array}$ & present & ve- & ve+ & $\begin{array}{l}\mathrm{LN} \text { and } \\
\text { peripencreatic }\end{array}$ \\
\hline 4 & 21 & $\mathrm{~F}$ & $\begin{array}{c}\text { Abdominal pain, fever, weight } \\
\text { loss }\end{array}$ & $\begin{array}{c}\text { Hypodense cystic septated lesion } \\
\text { between the liver inseparable from } \\
\text { pancreatic body }\end{array}$ & present & vet & ve+ & $\begin{array}{l}\text { liver, gall bladder, } \\
\text { stomach spleen, } \\
\text { omentum and } \\
\text { peritoneum }\end{array}$ \\
\hline 5 & 52 & $\mathrm{~F}$ & Abdominal pain, weight loss & $\begin{array}{l}\text { Well-defined heterogeneous mass in } \\
\text { the head and neck of the pancreas, } \\
\text { multiple LN }\end{array}$ & absent & ve- & ve+ & 1 \\
\hline 6 & 65 & $\mathrm{~F}$ & Chronic Abdominal pain & $\begin{array}{l}\text { Well-defined isodense mass in the } \\
\text { head of the panaceas, multiple } \\
\text { calcified LNs and mediastinal LNs }\end{array}$ & present & ve- & ve- & $\begin{array}{l}\mathrm{LN} \text { in chest and } \\
\text { abdomen, pancreatic }\end{array}$ \\
\hline 7 & 15 & M & $\begin{array}{l}\text { Abdominal pain, fever, SOB, } \\
\text { weight loss, cyanosis }\end{array}$ & $\begin{array}{c}\text { multiple abdominal lymph node are } \\
\text { noted at the gastro-splenic, porta } \\
\text { hepatis and at near the pancreatic } \\
\text { head. }\end{array}$ & present & ve- & ve- & chest, and pancreas. \\
\hline 8 & 59 & M & $\begin{array}{l}\text { Recurrent abdominal pain, } \\
\text { vomiting, weight loss }\end{array}$ & $\begin{array}{l}\text { Heterogeneous mass at head of } \\
\text { pancreas with calcification }\end{array}$ & present & ve- & ve- & $\begin{array}{c}\text { chest and abdomen, } \\
\text { pancreatic. }\end{array}$ \\
\hline 9 & 28 & M & Abdominal pain & $\begin{array}{l}\text { Peripancreatic homogeneously } \\
\text { hypovascular soft tissue lesion } \\
\text { with upper and mediastinal } \\
\text { lymphadenopathy. }\end{array}$ & present & ve- & ND & $\begin{array}{c}\text { chest and abdomen, } \\
\text { pancreatic. }\end{array}$ \\
\hline
\end{tabular}
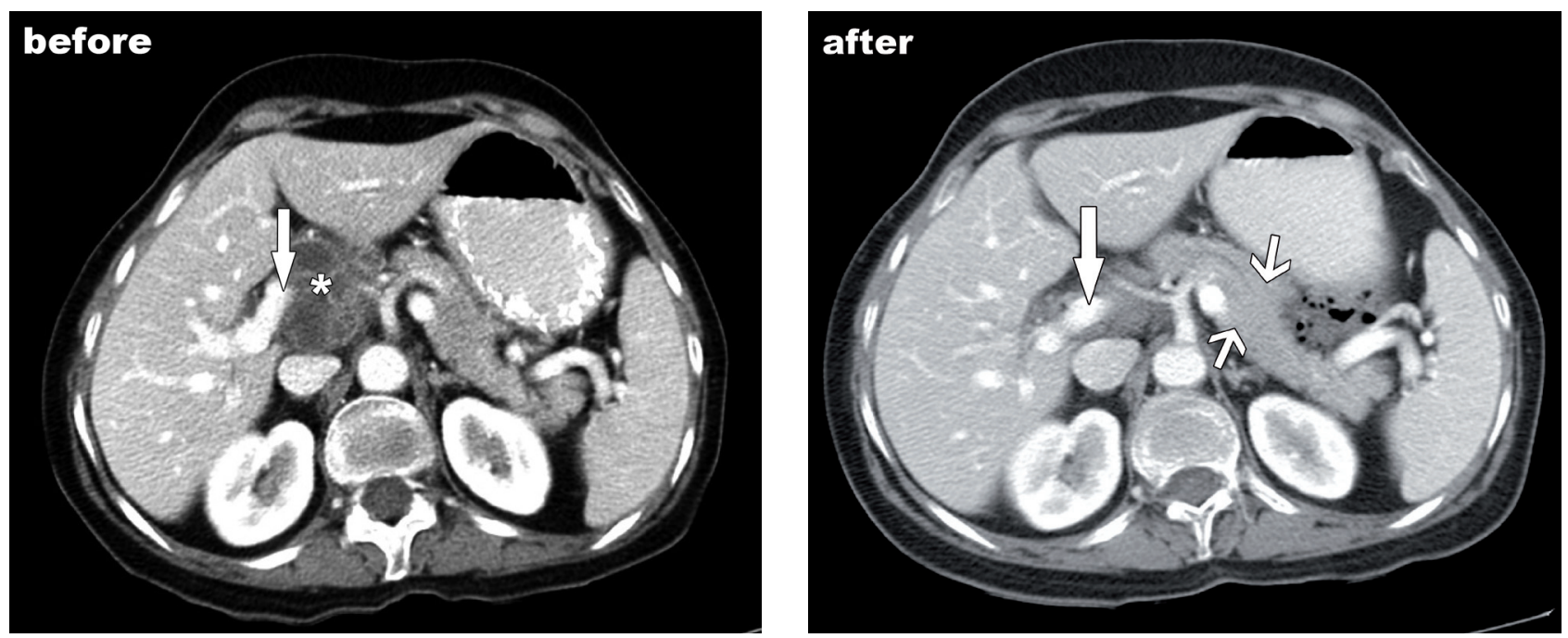

Figure 1 - Computed tomography of case 5 A) before and B) after. The white arrows - portal vein, opposing arrow heads - pancreas, asterisk $\left({ }^{*}\right)$ - portahepatic cyst like mass (necrotic lymphadenopathy due to tuberculosis).

Case 7. Patient started on rifampicin, INH, pyrazinamide, ethambutol, pyridoxine, vit B6. Followed up in the clinic, showed improvement.

Case 8. Patient started on rifampicin, INH, pyrazinamide, ethambutol, pyridoxine, vit B6. Followed up in the clinic, and showed improvement.
Case 9. Ethambutol, Pyrazinamide, Rifampicin, INH, vit B6. Followed up in the clinic, showed improvement.

All patients fully improved without significant side effects (except case no. 5) and showed good responses physically, clinically and radiologically (cases 5 and 9, shown in Figures $1 \& 2$ ). 

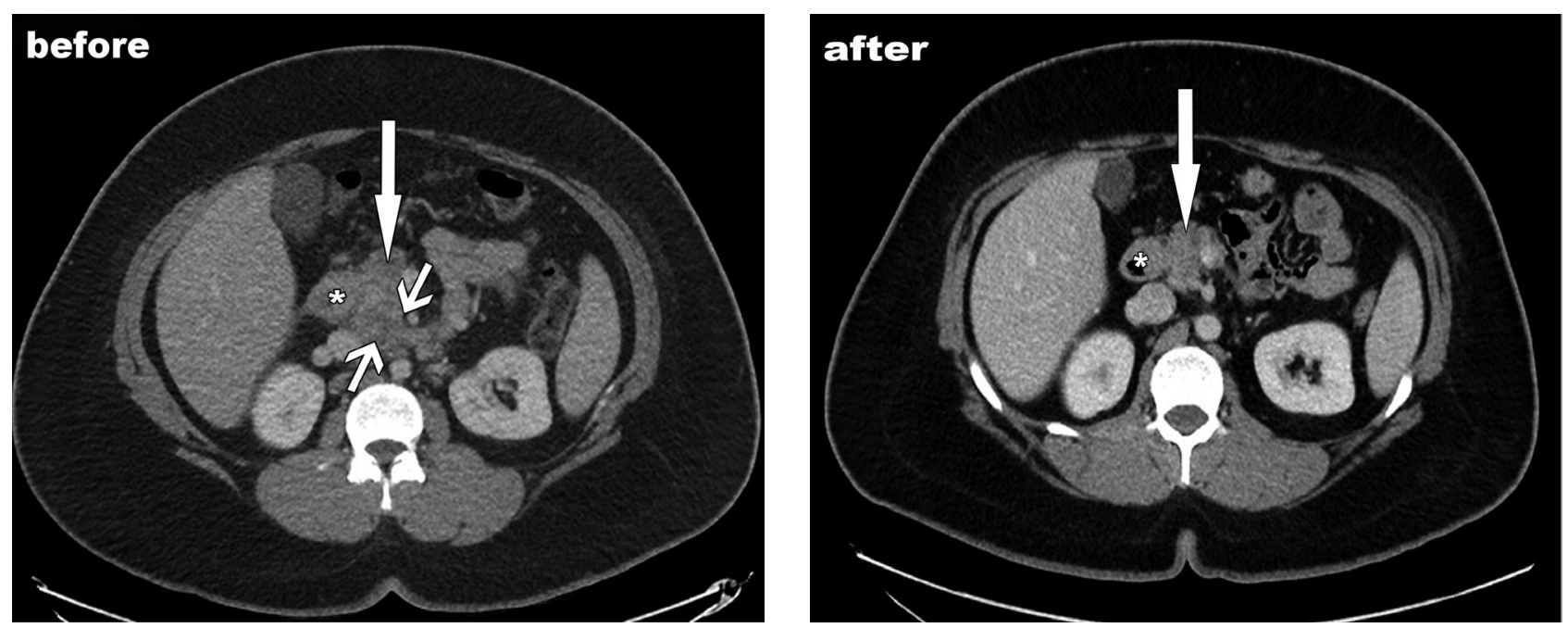

Figure 2 - Computed tomography of case 9 A) before and B) after. asterisk $\left({ }^{*}\right)$ - duodenum, arrow - dead of pancreas, opposing arrow-heads - hypodense matted lymphadenopathy tuberculosis.

Discussion. In this series, patients presented with variable signs and symptoms indicating involvement of many organs and most importantly pancreas with TB. Pancreatic TB is a rare entity which seldom affects the pancreas alone and focal pancreatic involvement without $\mathrm{TB}$ elsewhere in an immunocompetent patient is more rare. In western countries, there were also reports of confirmed increased frequency of pancreatic TB. The identified number of such cases in immunocompetent patients increased over the past decade. The increased occurrence is possibly brought in by globalization, increased use of immunosuppressants, the global resurgence of mycobacterium $\mathrm{TB}$ and the HIV pandemic. ${ }^{2}$

The presentation of common clinical features of pancreatic TB were unclear. Symptoms such as epigastric pain, weight loss, and low-grade fever are non-specific symptoms, which usually rise to the possibility of an abdominal malignancy. Pancreatic adenocarcinoma is the leading diagnosis in most reported cases of TB of the pancreas. Early detection is very significant to prevent redundant diagnostic or therapeutic procedures, since pancreatic TB is usually treated medically with excellent results. $^{3}$

Biopsies result from the mass showed high sensitivity of cultures $77 \%$ with antibiotic sensitivity but it can take up to 8 weeks to get the results, granulomas with or without caseation is sensitive in over $60 \%$ of cases and Ziehl-Neelsen stain is sensitive only in $50 \%$ of the cases. ${ }^{4}$

Fine needle aspiration cytology/biopsy is generally considered as a useful test and only few cases of pancreatic
TB have been diagnosed by FNA cytology/biopsy. The percentage of success of the image guided percutaneous FNA cytology or biopsy in diagnosing pancreatic TB is less than $50 \% .^{2}$ Endoscopic US-FNA cytology/biopsy has proven to be an excellent tool for the cytological diagnosis of pancreatic and peri pancreatic masses. The diagnostic accuracy of EUS-FNA was reported to be $76 \%$ to $95 \%$ for pancreatic cancer and $46 \%$ for focal inflammation. ${ }^{2}$ Because of the rarity of pancreatic/peri pancreatic TB, the diagnostic accuracy of EUS-FNA for this condition is difficult to determine. In a recent randomized controlled study, comparing EUS-guided biopsy and CT- or US-guided biopsy for determination of pancreatic mass etiology, no statistical difference was found in terms of accuracy. ${ }^{5}$ There is no known negative impact of tumor cell seeding with EUS-guided FNA of cystic or solid pancreatic masses. ${ }^{2}$

In our series, we have questionable cases suspected to be isolated pancreatic $\mathrm{TB}$, but we could not confirm it. Only 28 cases have been reported in the literature worldwide (1994). ${ }^{6}$ However, in a study from India between 1999-2004 detected pancreatic TB in 8.3\% of the 384 patients who were diagnosed with abdominal TB. ${ }^{7}$ Also, a study by Chaudhary, ${ }^{8}$ encountered 5 cases of pancreatic TB in the last 10 years. Out of these 5 cases, pancreatic TB was a part of generalized disease. ${ }^{9}$ In 1941, Auerbach ${ }^{10}$ reported autopsy results of 1656 TB patients. Pancreas was involved in 14 cases $(4.7 \%)$ as part of generalized TB but no isolated pancreatic TB cases were found. ${ }^{8}$ Bhansali ${ }^{11}$ did not report a single case of pancreatic TB in a series of 300 cases of abdominal tuberculosis. ${ }^{11}$ It was suggested that the 
pancreas is usually spared because of the presence of pancreatic enzymes, which interfere with the seeding of mycobacterium TB. ${ }^{12}$

Overall, the healthcare system in Saudi Arabia has improved dramatically and the government applied strict implementation of vaccination within the country. As such, physicians ruled out TB especially pancreatic in the differential diagnosis of any pancreatic mass.

In conclusion, in any patient presenting with a pancreatic mass suspicious for carcinoma, EUS and FNA are good tools to perform in order to confirm malignancy and rule out benign treatable conditions like TB.

Acknowledgment. The authors would like to thank Dr. Muteb Alkubaeer, Consultant, General Radiologist for attesting the clarity and correctness of the CT scan and Scribendi (www.scribendi.com) for editing the language.

\section{References}

1. Baraboutis I, Skoutelis A. Isolated tuberculosis of the pancreas. J Oncol Pract 2004; 5: 155-158.

2. Shahrokh S, Miri MB, Safari MT, Alizadeh AH. Pancreatic Tuberculosis: an overview. J Oncol Pract 2015 http:// pancreas.imedpub.com/pancreatic-tuberculosis-an-overview. php?aid $=4938 \# 10$

3. Ariga D. Pancreatic tuberculosis - not cancer. Infect Med 2002; 19: 566-750.
4. Song TJ, Lee SS, Park DH, Lee TY, Lee SO, Seo DW, et al. Yield of EUS-guided FNA on the diagnosis of pancreatic/ peripancreatic tuberculosis. Gastrointest Endosc 2009; 69: 484-491.

5. Chang KJ, Nguyen P, Erickson RA, Durnim TE, Katz KD. The clinical utility of endoscopic ultrasound-guided fine-needle aspiration in the diagnosis and staging of pancreatic carcinoma. Gastrointest Endosc 1997; 45: 387-393.

6. Wu CS, Wang SH, Kuo TT. Pancreatic tuberculosis mimicking pancreatic head carcinoma, a case report and review of the literature. Infection 1994; 22: 287-289.

7. Nagar AM, Raut AA, Morani AC, Sanghvi DA, Desai CS, Thapar VB. Pancreatic tuberculosis: a clinical and imaging review of 32 cases. J Comput Assist Tomogr 2009; 33: 136-141.

8. Chaudhary P, Bhadana U, Arora MP. Pancreatic tuberculosis. Indian J Surg 2015; 77: 517-524.

9. Singh DK, Haider A, Tatke M, Kumar P, Mishra PK. Primary pancreatic tuberculosis masquerading as a pancreatic tumor leading to whipple's pancreaticoduodenectomy. a case report and review of the literature. J Oncol Pract 2009; 10: 451-456.

10. Auerbach O. Acute Generalized miliary tuberculosis. Am J Pathol 1944 20: 121-36.

11. Bhansali SK. Abdominal tuberculosis. Experiences with 300 cases. Am J Gastroenterol 1977; 67:324-37

12. Ahlawat SK, Charabaty-Pishvaian A, Lewis JH, Haddad NG. Pancreatic tuberculosis diagnosed with endoscopic ultrasound guided fine needle aspiration. J Oncol Pract 2005; 6: 598-602.

\section{Copyright}

Whenever a manuscript contains material (tables, figures, etc.) which is protected by copyright (previously published), it is the obligation of the author to obtain written permission from the holder of the copyright (usually the publisher) to reproduce the material in Saudi Medical Journal. This also applies if the material is the authors own work. Please submit copies of the material from the source in which it was first published. 